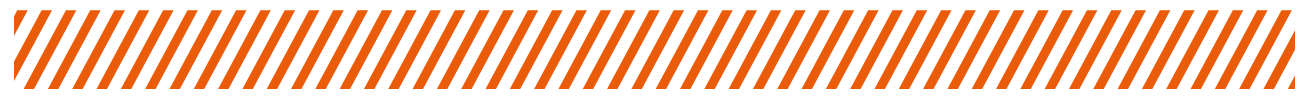

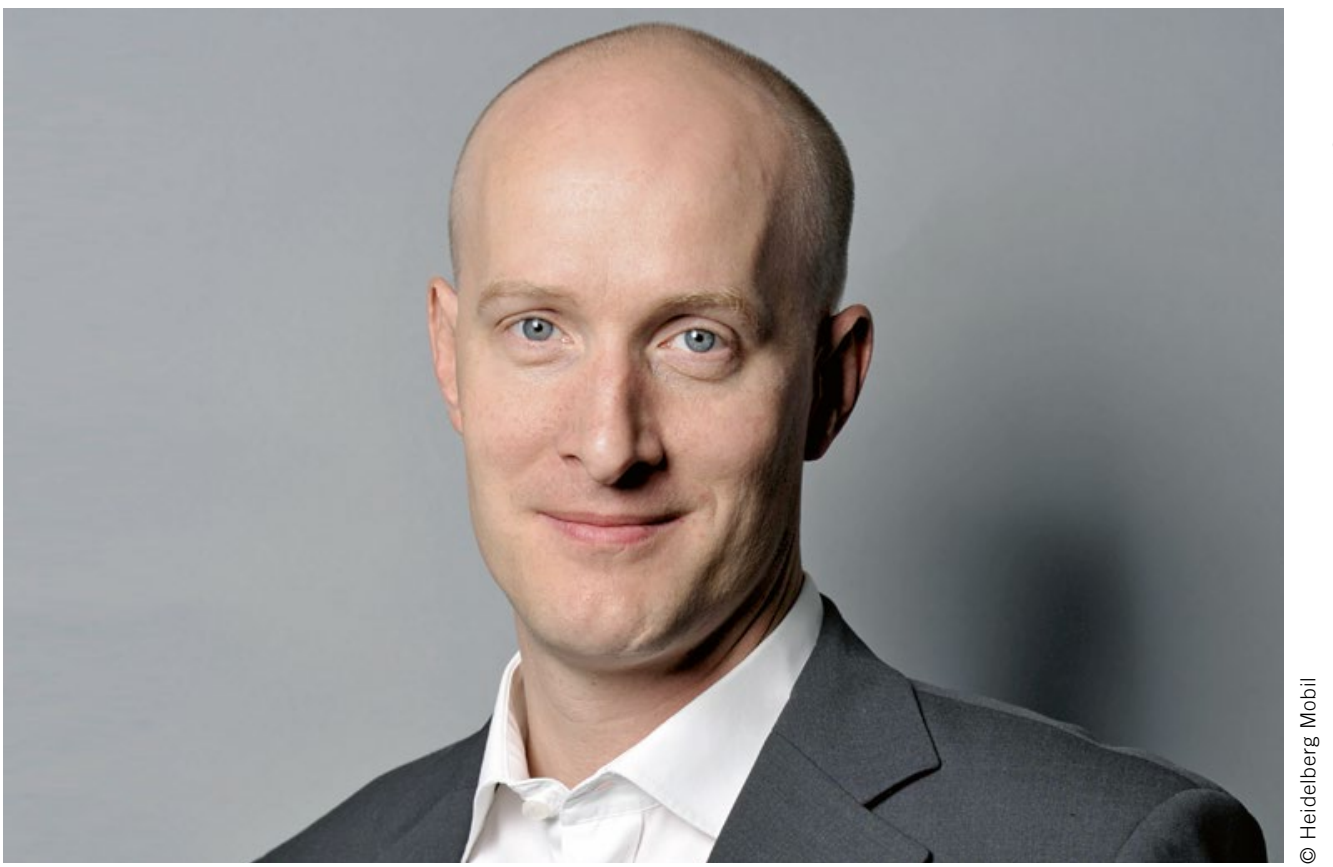

Dr. rer. nat. Matthias Jöst Komiteeleiter omlox bei der Profibus Nutzerorganisation e. V. in Kalsruhe und Geschäftsführer der Heidelberg Mobil International $\mathrm{GmbH}$ in Heidelberg

\section{Standardisierte Ortungsdaten für die Produktion und Logistik}

Im Zeichen von Covid-19 und Social Distancing haben uns die vergangenen Monate die eminente Bedeutung von Transparenz in der Lieferkette aufgezeigt. Aber nicht nur für das Gemeinwesen ist das Wissen vom Wo von hoher Relevanz, sondern auch in der industriellen Produktion werden ortsbasierte Daten zu einem elementaren Rohstoff.

Eine modulare Produktion, der autonome Werksverkehr, transparente Lieferketten, nachhaltige Wertstoffströme, effiziente Nutzung von Betriebsmitteln, die Gewährleistung der Mitarbeitersicherheit oder der Aufbau von nutzungsbasierten Geschäftsmodellen - die Liste der Anwendungsfälle in denen universell verfügbare Ortungsdaten ein elementarer Enabler sind, ist sehr lang. Gelingt es heute außerhalb von Gebäuden sehr leicht, über Satelliten-gestützte Systeme standardisierte Ortungsdaten von Fahrzeugen, Gütern, Maschinen oder Personen zu erhalten, stellt sich das innerhalb von Gebäuden - in dem ein Großteil der Produktion und Wertschöpfung stattfindet - jedoch noch ganz anders dar. RFID, Bluetooth, WiFi, Ultra-Wide-Band, SLAM - ein großes Repertoire an technischen Verfahren erlaubt es, in Logistik- und Produktionsprozessen Ortungsdaten zu generieren. Stand heute ist allerdings keines der Verfahren dazu geeignet, für alle räumlichen und technischen Gegebenheiten, Ortungsdaten in gleicher Qualität und Aktualität bereitzustellen.
Dieser Umstand hat zur Folge, dass die Ortungslandschaft der Automobilproduktion durch einen Flickenteppich an Insellösungen und Datensilos gekennzeichnet ist. Einige OEMs haben diese Problemstellung für sich erkannt und in den vergangenen Jahren für ihre Produktionsstandorte in den Aufbau eigener Ortungsdatenplattformen investiert. Die breite Masse der Zulieferer bleibt hier jedoch noch außen vor und kann nur mit Insellösungen Ortungsdaten zur Steigerung der eigenen Wettbewerbsfähigkeit einsetzen.

Genau diesem Umstand hat sich der mit dem Hermes Award 2020 ausgezeichnete Ortungsstandard omlox - the open locating standard angenommen. omlox integriert alle gängigen Ortungstechnologien und stellt Ortungsdaten in einem definierten Datenformat systemübergreifend bereit. Zudem erlaubt er die Interoperabilität von Trackern unterschiedlicher Hersteller. Damit wird auch der Mittelstand in die Lage versetzt, Produktions- und Logistikprozesse mithilfe von Ortungsdaten zu optimieren. Getragen wird omlox durch ein breites, herstellerübergreifendes Ökosystem, mit den Schwerpunkten Datenintegration und Geräteinteroperabilität für Ortung in industriellen Anwendungsfällen. Als offener Standard und Teil des Profibus Nutzerorganisation e. V. sind alle Unternehmen eingeladen, an der Weiterentwicklung von omlox mitzuwirken. 